\title{
Analytical Study and Numerical Solutions of Bird Flu Epidemic Model with Vaccination
}

\author{
Arrival Rince Putri ${ }^{1}$, Mahdhivan Syafwan ${ }^{2}$ \\ \{arrivalputri@gmail.com ${ }^{1}$ \} \\ Graduate School Environmental and Life Science, Okayama University, Japan ${ }^{1}$
}

\begin{abstract}
Mathematical modeling is a tool used for the mechanism of strategy in controlling epidemics. In this paper, a basic model for bird flu epidemic is developed. Vaccination treatment of bird flu is a common method used to control disease. The bird flu model is reformulated by taking into account vaccination. Those models are system of ordinary differential equations. Stability of both the models are discussed and associated with the threshold number stating population is free of bird flu virus or infected. Numerical solutions are given on phase plane to confirm the analytical results.
\end{abstract}

Keywords: Epidemic model, Vaccination, Stability, Threshold number, Numerical solutions

\section{Introduction}

Bird flu or avian influenza is a disease transmitted from birds to humans. Two types of avian influenza viruses, namely H5N1 and H7N9. The virus is an influenza virus that attacks both wild birds and poultry farm, such as chicken, duck, geese, or other birds. If it is given the opportunity to infect together with influenza viruses in humans, then infectious diseases in humans will emerge and it begin spreading from human to human. Moreover, bird flu is transmitted through direct contact with infected birds or contaminated environment.

Transmission of the bird flu virus is difficult to prevent. But it can be prevented by avoiding contact with surfaces contaminated with wild birds or domestic birds faeces. Another way of its prevention is by vaccination. Vaccination is one of commonly used method against infectious disease. Vaccination is very important to control the spread of bird flu. The level of vaccination against an individual determines the level of population immunity and the possibility of an epidemic occur.

A number of mathematical models discussing the spread of bird flu have been previous studied. Mathematical models for simulating the process of bird flu infection have been discussed and analyzed [1-4]. Stability analysis of susceptible populations with vaccination in the SIR model is Discussed [5]. Analysis of the model associated with varying vaccination strategies for the Avian Flu virus (H5N1) on chicken populations is studied [6]. A transmission model of the Avian influenza disease was developed and analyzed by optimization vaccination and medical treatment [7]. A bird flu model with vaccination considering constant population is analyzed [8].

In this study, two types of epidemic models of the spread of bird flu are analyzed, that are model without vaccination and model with vaccination. Those model are expressed in the form of an ordinary differential equation. The models are developed based on the basic SIR 
(Susceptible Infected Recovered) model. Behaviour of the bird flu epidemic will be known when vaccination policies are implemented by analyzing the model.

\section{Methods}

First, some facts and assumptions from various related literature are studied and completed to develop the model. Second, the basic SIR model is reformulated with and without vaccination. Critical points and their stability of those model are analyzed. Threshold number corresponded with the equilibrium point are determined. Last section, numerical simulations for solution of the model with some parameter value are given.

\section{Mathematical Models}

The spread of bird flu in the population is divided into three groups, that are susceptible (healthy individuals but can be infected), infected (sick individuals infected by a virus), and removed (healed and die individuals). The model is developed under the following some assumptions:

- The system is an open system.

- All newborn individuals are into the susceptible group.

- There is no infected individual becoming susceptible again

- An individual can leave the infected group by being infected, and it can leave can the infected group by removing.

- The death rate is equal for all individuals in the three groups.

- Vaccination is given to all newborn individuals.

Base on those assumptions, the bird flu epidemic model without vaccination leads to the following system of Equation (1)

$$
\begin{aligned}
& \frac{d S}{d t}=\mu-\beta S I-\tau S, \\
& \frac{d I}{d t}=\beta S I-\tau I-\gamma I, \\
& \frac{d R}{d t}=\gamma I-\tau R,
\end{aligned}
$$

Where $S$ is the number of susceptible individuals, $I$ is the number of infected individuals, and $R$ is the number of removed individuals. Parameter $\mu$ is the birth rate, $\beta$ is the contact rate of virus transmission, and $\tau$ is the death rate, and $\gamma$ is the recovery rate. All parameters are positive constant.

The first two equations of the system (1) is

$$
\begin{aligned}
& \frac{d S}{d t}=\mu-\beta S-\tau S, \\
& \frac{d I}{d t}=\beta S I-\tau I-\gamma I,
\end{aligned}
$$


Domain of the system (2) in Equation (2) is defined by $\Psi_{1}=\left\{D_{1} \in R^{2} \mid D_{1}=(S, I)\right.$, where $S$ $\geq 0$ and $I \geq 0\}$. The transmission of bird flu virus in the population can be inhibited by giving vaccinations to all newborn individuals. In this paper, we assumed that all newborn individuals are vaccinated with vaccination rate between $0<\rho<1$. Base on this assumption, the system (2) becomes to the following system of Equations (3)

$$
\begin{aligned}
& \frac{d S}{d t}=(1-\rho) \mu-\beta S I-\tau S, \\
& \frac{d I}{d t}=\beta S I-\tau I-\gamma I,
\end{aligned}
$$

Where parameter $\rho$ is the vaccination rate. Stability of the system (2) and (3) will be discussed in the next following section.

\section{Stability Analysis}

\subsection{Model without vaccination}

There are two equilibrium points of the system (2) that correspond with two states

- $\quad$ Bird flu-free state, $E_{0}=\left(\frac{\mu}{\tau}, 0\right)$.

- Endemic state, $E_{1}=\left(S_{0}, I_{0}\right)$, where $S_{1}=\frac{\tau+\gamma}{\beta}$, and $I_{1}=\frac{\mu \beta-\tau(\tau+\gamma)}{\beta(\tau+\gamma)}$.

Define threshold number is as like Equation (4)

$$
R_{0}=\frac{\mu \beta}{\tau(\tau+\gamma)}
$$

The endemic equilibrium $E_{1}$ exists if and only if $R_{0}>1$.

Theorem 1. The bird flu-free equilibrium point $E_{0}$ of the system (2) is locally asymptotically stable if only if $R_{0}<1$, and other is unstable.

Proof. Jacobian matrix at $E_{0}$ is like in Equation (5).

$$
J\left(E_{0}\right)=\left[\begin{array}{cc}
-\tau & -\frac{\mu \beta}{\tau} \\
0 & \frac{\mu \beta-\tau(\tau+\gamma)}{\tau}
\end{array}\right]
$$

Eigenvalues of $J\left(E_{0}\right)$ are $-\tau$ and $\frac{\mu \beta-\tau(\tau+\gamma)}{\tau}$. All eigenvalues are negative if and only if $R_{0}<1$. It means that the bird flufree equilibrium point $E_{0}$ is asymptotically stable. This proves Theorem 1.

Theorem 2. The endemic equilibrium point $E_{1}$ of the system (2) is locally asymptotically stable if only if $R_{0}>1$, and other is unstable.

Proof. Jacobian matrix at $E_{1}$ is Equation (6). 


$$
J\left(E_{1}\right)=\left[\begin{array}{cc}
-\frac{\mu \beta}{\tau+\gamma} & -\tau-\gamma \\
\frac{\mu \beta-\tau(\tau+\gamma)}{\tau+\gamma} & 0
\end{array}\right]
$$

The characteristic equation of $J\left(E_{0}\right)$ is Equation (7).

$$
\lambda^{2}+\left(\frac{\mu \beta}{\tau+\gamma}\right) \lambda+\left(\mu \beta-\tau^{2}-\tau \gamma\right)
$$

Eigenvalues of $J\left(E_{1}\right)$ are Equation (8).

$$
\begin{aligned}
\lambda_{1,2} & =\frac{-\frac{\mu \beta}{\tau+\gamma} \pm \sqrt{D_{0}}}{2} \\
& =\frac{1}{2}\left(-\tau R_{0} \pm \sqrt{D_{0}}\right)
\end{aligned}
$$

Where $D=\left(\tau R_{0}\right)^{2}-4(\mu \beta-(\tau+\gamma))$. Eigenvalues $\lambda_{1}$ and $\lambda_{2}$ depend on value of under the square root. If $D_{0}>0$, then there are two negative real eigenvalues with condition $\sqrt{D_{0}}$ must be smaller than $\tau R_{0}$. If $D_{0}<0$, then there are complex eigenvalues with the real part is negative, $-\tau R_{0}$. All eigenvalues are negative if only if $R_{0}>1$. It means that the endemic equilibrium point $E_{1}$ is asymptotically stable. This proves Theorem 2 .

\subsection{Model with vaccination}

There are two equilibrium points of the system (3) that correspond with two states

- Bird flu-free state, $E_{\mathrm{v} 0}=\left(\frac{(1-\rho) \mu}{\tau}, 0\right)$.

- Endemic state, $E_{\mathrm{v} 1}=\left(S_{\mathrm{v}}, I_{\mathrm{v}}\right)$, where $S_{\mathrm{v}}=\frac{\tau+\gamma}{\beta}$, and $I_{\mathrm{v}}=\frac{(1-\rho) \mu \beta-\tau(\tau+\gamma)}{\beta(\tau+\gamma)}$.

Define threshold number is as like Equation (9)

$$
R_{\mathrm{v}}=\frac{(1-\rho) \mu \beta}{\tau(\tau+\gamma)}=(1-\rho) R_{0}
$$

The endemic equilibrium $E_{\mathrm{v} 1}$ exists if and only if $R_{\mathrm{v}}>1$.

Theorem 3. The bird flu-free equilibrium point $E_{\mathrm{v} 0}$ of the system (3) is locally asymptotically stable if only if $R_{\mathrm{v}}<1$, and other is unstable.

Proof. Jacobian matrix at $E_{\mathrm{v} 0}$ is Equation (10).

$$
J\left(E_{v 0}\right)=\left[\begin{array}{cc}
-\tau & -\frac{\mu \beta(1-\rho)}{\tau} \\
0 & \frac{\mu \beta(1-\rho)-\tau(\tau+\gamma)}{\tau}
\end{array}\right]
$$

Eigenvalues of $J\left(E_{v 0}\right)$ are $-\tau$ and $\frac{\mu \beta(1-\rho)-\tau(\tau+\gamma)}{\tau}$. All eigenvalues are negative if and only if $R_{\mathrm{v}}<1$. It means that the bird flu-free equilibrium point $E_{\mathrm{v} 0}$ is asymptotically stable. This proves Theorem 3. 
Theorem 4. The endemic equilibrium point $E_{\mathrm{v} 1}$ of the system (3) is locally asymptotically stable if only if $R_{\mathrm{v}}>1$, and other is unstable.

Proof. Jacobian matrix at $E_{\mathrm{v} 1}$ is Equation (11).

$$
J\left(E_{\mathrm{v} 1}\right)=\left[\begin{array}{cc}
-\frac{\mu \beta(1-\rho)}{\tau+\gamma} & -\tau-\gamma \\
\frac{\mu \beta(1-\rho)-\tau(\tau+\gamma)}{\tau+\gamma} & 0
\end{array}\right]
$$

The characteristic equation of $J\left(E_{\mathrm{v} 1}\right)$ is Equation (12)

$$
\lambda^{2}+\left(\frac{\mu \beta(1-\rho)}{\tau+\gamma}\right) \lambda+\left(\mu \beta(1-\rho)-\tau^{2}-\tau \gamma\right)
$$

Eigenvalues of $J\left(E_{\mathrm{v} 1}\right)$ are Equation (13)

$$
\begin{aligned}
\lambda_{1,2} & =\frac{-\frac{\mu \beta(1-\rho)}{\tau+\gamma} \pm \sqrt{D_{\mathrm{v}}}}{2} \\
& =\frac{1}{2}\left(-\tau R_{\mathrm{v}} \pm \sqrt{D_{\mathrm{v}}}\right)
\end{aligned}
$$

Where $D_{\mathrm{v}}=\left(\tau R_{0}\right)^{2}-4(\mu \beta(1-\rho)-\tau(\tau+\gamma))$. Eigenvalues $\lambda_{1}$ and $\lambda_{2}$ depend on value of under the square root. If $D_{\mathrm{v}}>0$, then there are two negative real eigenvalues with condition $\sqrt{D_{v}}$ must be smaller than $\tau R_{\mathrm{v}}$. If $D_{\mathrm{v}}<0$, then there are complex eigenvalues with the real part is negative, $-\tau R_{\mathrm{v}}$. All eigenvalues are negative if only if $R_{\mathrm{v}}>1$. It means that the endemic equilibrium point $E_{\mathrm{v} 1}$ is asymptotically stable. This proves Theorem 4.

\section{Numerical Solutions}

The analytical results will be discussed by taking a numerical examples. By letting the parameters $\mu=0.5, \tau=0.3, \beta=1.98, \gamma=0.5$ and $\rho=0.6$, graphic solutions and portrait phase of the system (2) and the system (3) are given. Figure $\mathbf{1}$ and Figure $\mathbf{2}$ show table that the endemic equilibrium point is stable. The susceptible and infected populations will survive in the same time and the trajectory will approach the endemic equilibrium point. Figure 3 and Figure 4 show that the effect of vaccination can be seen on the bird flu-free equilibrium point and endemic equilibrium point. The susceptible population has decreased by vaccinating with vaccination rate $\rho$. 


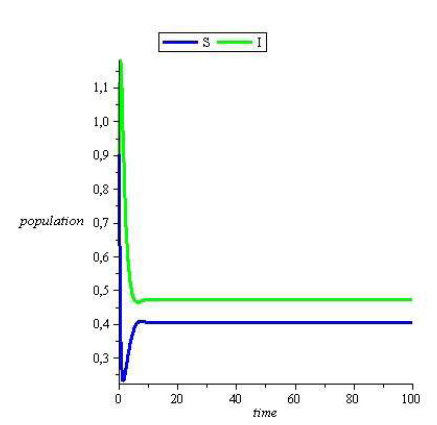

Fig. 1. Solutions of the system (2).

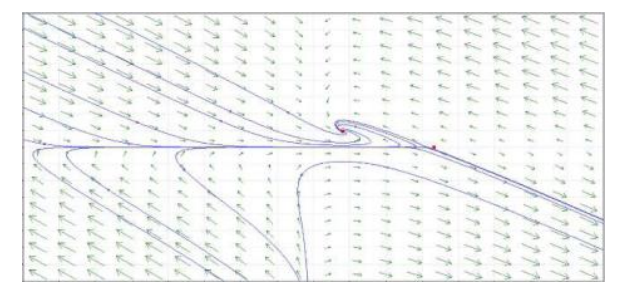

Fig. 2. Portrait phase of the system (2).

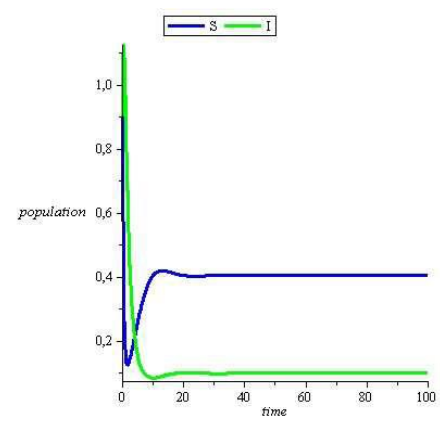

Fig. 3. Solutions of the system (3).

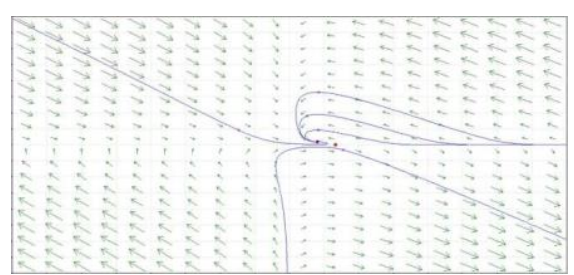

Fig. 4. Portrait phase of the system (3).

\section{Conclusion}

In this paper we study epidemic models of infectious diseases, bird flu. The two epidemic models, without and with vaccination, are compared. The characteristics of those models given the stability associated with the thresholds number. It can be conclude that vaccination more effective to inhibiting the transmission of the bird flu virus effectively than without vaccination.

Acknowledgments. Author would like acknowledge to the Directorate General of Higher Education, Republic of Indonesia for the funding of this research.

\section{Authors' Note}

The author(s) declare(s) that there is no conflict of interest regarding the publication of this article. Authors confirmed that the paper are free of plagiarism.

\section{References}

[1] Iwami, S., Takeuchi, Y., \& Liu, X.: Avian-human influenza epidemic model. Mathematical Bioscience. Vol. 207.pp. 1-25 (2005)

[2] Rao, D.M., Chernyakhosvsky, A., \& Rao, V.: Modeling and analysis of global epidemiology of avian influenza. Environmental Modeling and software. Vol. 24. pp. 124-134 (2009)

[3] Derouich, M. and Boutayeb, A.: An avian influenza mathematical model. Applied Mathematical Science. Vol. 2 No. 36. pp. 1749-1760 (2008) 
[4] Kimbir, A.R., Aboiyar, T., \& Okolo, P.N.: A model analysis for the transmission dynamics of avian influenza. Mathematical Theory and Modeling. Vol. 4 No. 13. pp. 15-28 (2014)

[5] Zaman, G., Kang, Y.H., and Jung, I.H.: Stability analysis and optimal vaccination of an SIR epidemic model. Biosystems. Vol. 93. No. 3. pp. 240-249 (2008)

[6] Galvin, C.J., Rumbos, A., Vincent, J.I., \& Salvato, M.: Modeling the effects of avian flu (H5N1) vaccination strategies on poultry. CODEE Journal. Vol. 10. pp. 1-10 (2014)

[7] Tasmi, C.J., \& Nuraini, N.: Optimal vaccination and treatment schedules in a deterministic avian influenza model. J. Math. Fund. Sci.. Vol. 48 No. 2. pp. 164-177 (2016)

[8] Kharis, M., \& Amidi.: Mathematical modeling of Avian Influenza epidemic with bird vaccination in constant population. J. Phys.: Conf. Ser. Vol. 983. pp. 1-9 (2018) 